\title{
Intelligent CAD system for the Hydraulic Manifold Blocks
}

\author{
Jinwei Bai ${ }^{1}$, Guang $\mathrm{Li}^{1}$, Junqiang Wang ${ }^{1}$, Weiguo Gao ${ }^{1}$, and Wentie Niu ${ }^{1}$ a \\ ${ }^{1}$ Key Laboratory of Mechanism Theory and Equipment Design of The State Education Ministry, \\ Tianjin University, Tianjin 300350, China \\ aniuwentie@tju.edu.cn
}

Keywords: Hydraulic Manifold Blocks, layout and connection design, intelligent design, CAD. Abstract. In order to improve the design efficiency and quality of hydraulic manifold blocks, architecture of intelligent CAD system for hydraulic manifold blocks was proposed. A component layout rule library and an oil circuit connection pattern library were established to integrate empirical knowledge, design expertise, design standard and code. For the components layout design, a core components based layout design method for hydraulic components was proposed, which can decrease reduce the design complexity of main oil circuits. For connection design, a hole-arrangement design approach was proposed by combining pattern matching of typical topology connection oil circulation and intelligent path planning algorithm, which can improve the design efficiency by reusing design knowledge of oil circuits. Intelligent CAD system for the hydraulic manifold blocks was developed by using SolidWorks Application Programming Interface (API). A design example is given to illustrate the feasibility and efficacy of the proposed framework.

\section{Introduction}

The interactions between the external components layout and internal connection design are the major problem $[1,2]$ that greatly increase the design complexity of hydraulic manifold blocks. Intelligent optimization method, such as genetic algorithm [3], evolutionary programming [4] and simulated annealing algorithm [5] is one solution for the layout and connection design problem of HMB. However, pure computational intelligence based design optimization of HMB may result in low speed of solving, poor design relevance. And complex engineering constraints, such as installation, adjustment and maintenance of components, cannot be taken into consideration.

Intelligent CAD system, which combines knowledge based design and intelligent optimization, is the trend to solve the layout and connection design problem of HMB. In order to improve the efficiency and rationality of cartridge manifold block design, Zhang [6] proposed a parametric design approach for layouts of external hydraulic components and a heuristic algorithm for hole arrangements to optimize connection sequences of internal holes. Cheng [7] proposed a CAD system structure to improve the design efficiency and overall performance of manifold block. In their proposed design system, a back tracking algorithm based on engineering rules is put forward and a multi-hole routing approach is proposed to improve the design efficiency.

Based on the systematically summarizing design standard and code, empirical knowledge and design expertise, and product manual of hydraulic manifold blocks, a design knowledge base, including component layout rule of component and typical oil circuits connecting pattern, is established. On the basis of components layout approach [8], an oil circulation connection design approach was proposed by combining pattern matching of typical oil circulation and intelligent path planning algorithm. Based on the aforementioned work, an intelligent CAD system for the hydraulic manifold blocks is developed by utilizing the SolidWorks API, VB.NET and Matlab.

\section{Architecture of intelligent design system for hydraulic manifold blocks}

Fig. 1 illustrates the architecture of intelligent design system for hydraulic manifold blocks. It consists of five modules, including design information input module, component layout module, connection design module, interference check module, and design knowledge management module. 
Design information input module obtains the necessary information of hydraulic element and connection relation from the hydraulic schematic, retrieves corresponding hydraulic element from the library, and then assemble them on the block. Component layout module determines the installing surface and the location of components by utilizing rule-based reasoning. Connection design module obtains the detailed information of connected ports of oil circuits according to the connection relation firstly. Then, pattern matching algorithm or intelligent path planning algorithm is performed to obtain the detailed parameters of holes arrangement. Finally, the parametric CAD model of oil circuit is generated by utilizing SolidWorks API. Interference check module includes three sub-modules. Component interference checking function of SolidWorks is performed to determine if the interference of hydraulic elements is occurred. The minimum distance of any two oil circuits is calculated to check the correctness of connection relation and minimum wall thickness among them. Design knowledge management module manages all the design information and supporting databases, including design data and parametric assembly model of HMB as well as hydraulic elements library, rule library, and typical connection pattern library.

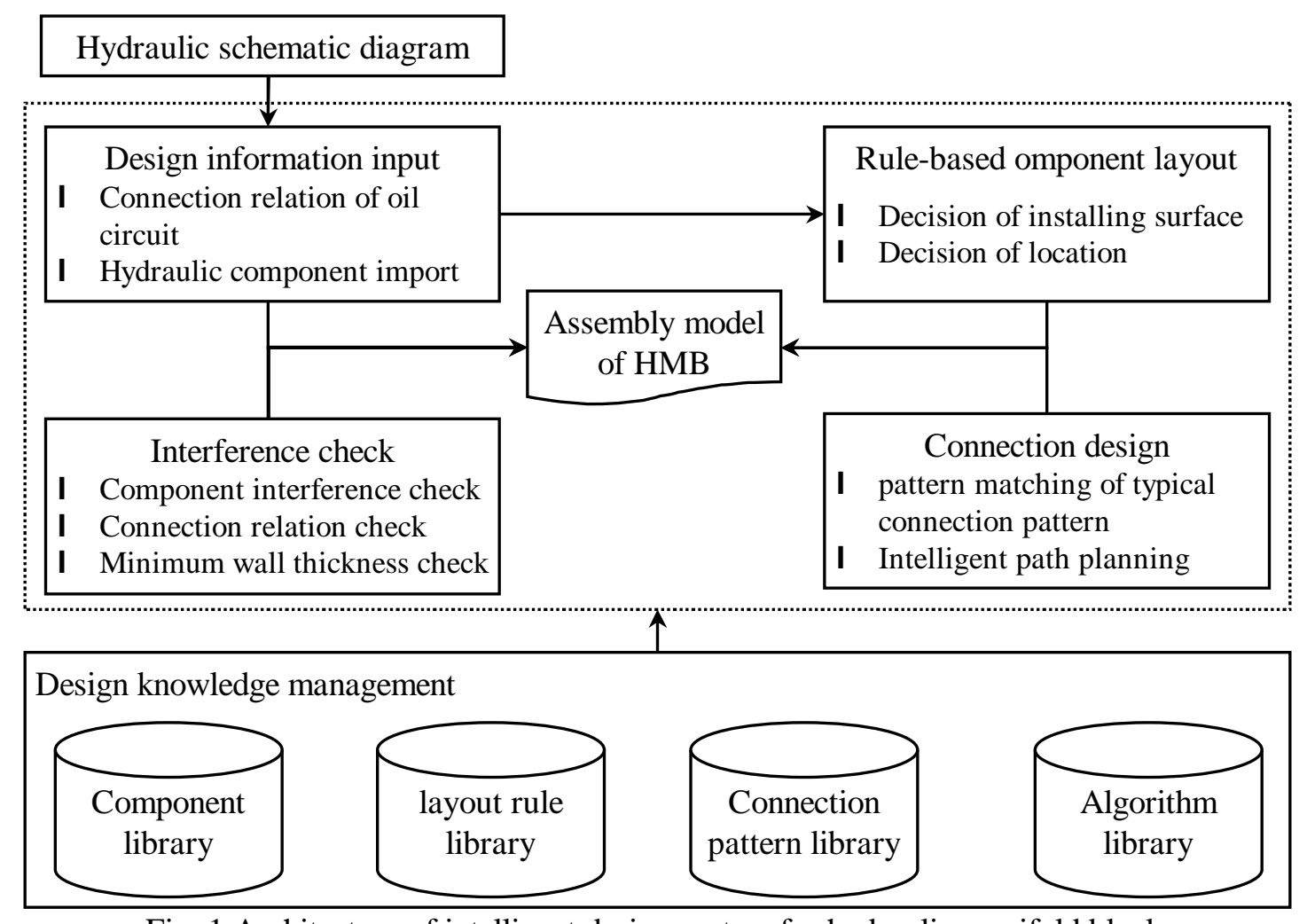

Fig. 1 Architecture of intelligent design system for hydraulic manifold block.

\section{Layout and connection design}

Description of design knowledge. Object-oriented model is used to store and transmit the design information, manage design knowledge and product models as well as design process. Class Component encapsulates the basic attributes, the dimensions and other data relating to mounting surface or cavity for hydraulic elements. Class OliCirculation encapsulates parameters of each hole of an oil circuit and their geometric modeling knowledge as well as design constraints. It also manages the process of connection design. To facilitate interference check, temporary body model of SolidWorks is used to represent screw holes, pin holes and cavity of component and the oil holes of circuits, as shown in Fig. 2.

Design knowledge is the core of the intelligent design system for hydraulic manifold block, which directly influences the legitimacy of the design result. Generally, design knowledge includes empirical knowledge, design expertise, design standard and code as well as model knowledge of hydraulic 
component. Component library includes parametric CAD model, mounting and location datum as well as interface data. For empirical knowledge, design expertise, design standard and code, are represented by production rules and typical connection pattern of oil circulation.
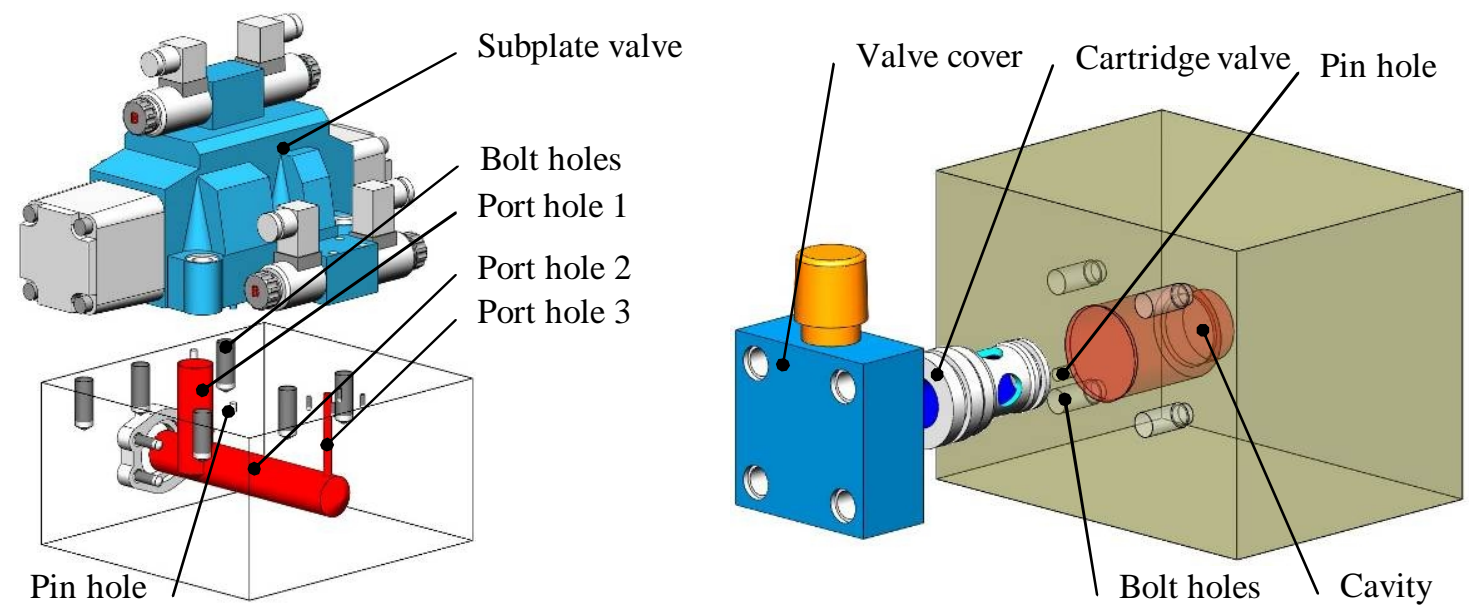

Fig. 2 Components and oil circuit.

Rule-based component layout. The installation surface of the hydraulic element in the manifold block greatly affects the design of hydraulic manifold blocks. So the primary task of component layout is to determine the installation surface. Some rules [8] which determine the installation surface are summarized as follows:

(1) Important and larger size components on up face (face 5) of block.

(2) Adjustable components on front face (face 1) for operation convenience.

(3) Components connected to ports $\mathrm{P}$ and $\mathrm{T}$ on left face (face 2) or right face (face 4).

(4) Components connected to ports X, Y and L on left face (face 2) or right face (face 4).

(5) Components connected to ports A and B on back face (face 3).

(6) Cartridge valves on front face (face 1) or up face (face 5).

(7) Bottom face (face 6) is the mounting surface of block.

The basic idea of layout design of hydraulic element is depicted in Fig. 3. In this paper, plate valve which contains ports $\mathrm{P}, \mathrm{T}, \mathrm{A}$ and $\mathrm{B}$ is defined as core component. According to principles, core components are placed on face 5. As shown in Fig. 3(a), the location of core component is determined by parameters $L 1, L 2$, and $L 3$. The arrangement of rest components is depicted in Fig. 3(b). The installation surfaces of the rest components are determined by the aforementioned layout principles. As shown in Fig. 3(b), the locations of rest components can be determined by setting the corresponding values of parameters $H 1, H 2, H 3, H 4, L 5$ and $L 6$. By systematically summarizing the expert experience, successful design case, design standard and code, the values of these parameters can be extracted to form the layout design knowledge of hydraulic elements.

The layout procedures are as follows. Firstly, core components are determined according to the hydraulic schematic. Secondly, the components are divided into several groups. In this step, all the core components are assigned to a separate group; components which have connection relation with different core component are assigned to different group; components which have no connection relation with any core component are assigned to a group. Then, groups are sorted according to the nominal diameter of core component. Thirdly, design sequence of oil circuit is determined according to the diameter and the pressure of oil circuit. The order of pressure is $\mathrm{P}, \mathrm{A}, \mathrm{B}, \mathrm{T}, \mathrm{X}, \mathrm{Y}, \mathrm{L}$. Then, the layout sequence of components can be determined according to both the component group and the design sequence of oil circuit. Finally, layout rules for each component are retrieved, the installation surface and location of each component are determined according to the layout rules. 


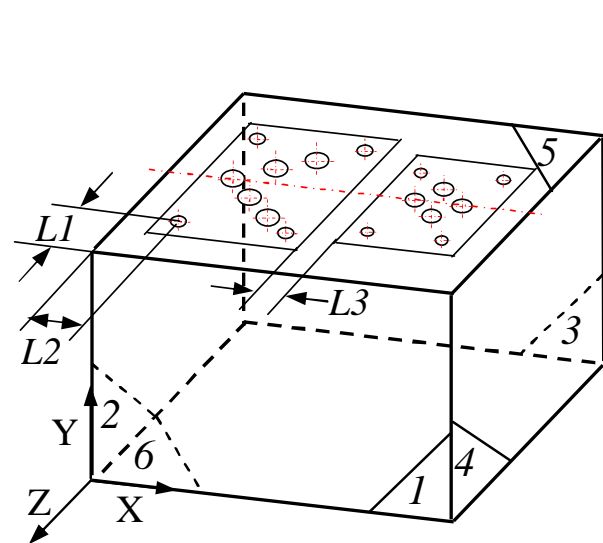

(a

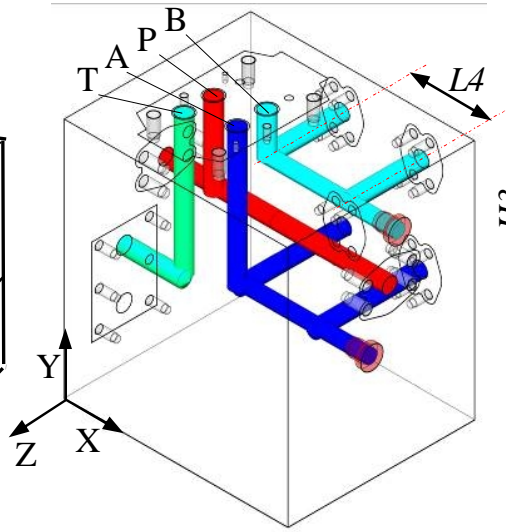

(b)

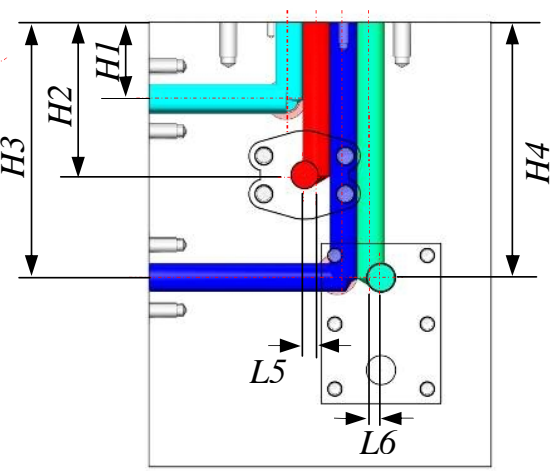

(b)

Fig. 3 Layout principle of components.

Connection design. Design order of oil circulation greatly affects the design results [7]. In general, connection design of main circuits should be a priority. As shown in Fig. 3, the holes of main circuits are arranged at different layer according to the layout of component. This means the complexity of connection design of main circuits have been reduced considerably. By systematically summarizing the expert experience, successful design case, design standard and code, typical connection patterns of main circuits are extracted and library of typical connection pattern is established. Connection design of main circuits can be achieved by matching of an appropriate circuit connection pattern. Some of typical connection pattern of two-port and multi-port circuits are shown in Fig. 4 and Fig. 5 respectively.

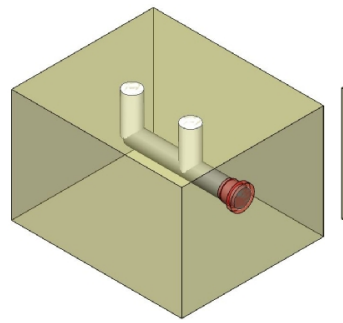

(a) Same face

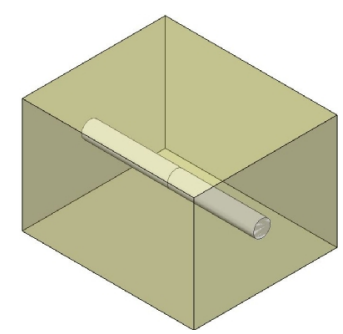

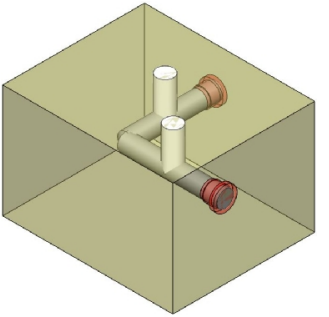

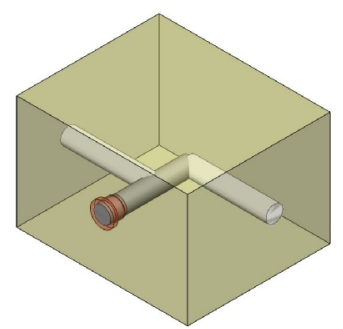

(c) Opposite face
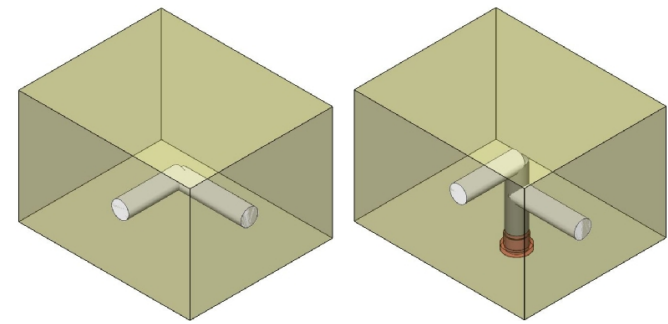

(b) Adjacent face

Fig. 4 Typical connection pattern of two-port oil circuit.

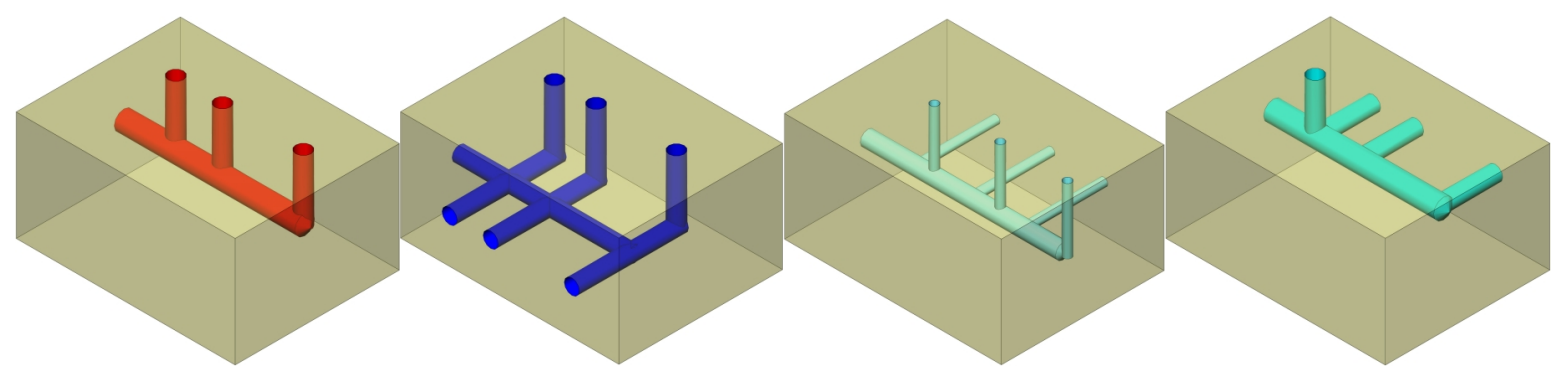

Fig. 5 Typical connection pattern of multi-port oil circuit. 
Connection design process of multi-port oil circulation is shown in Fig. 6. Firstly, the type of connection pattern of oil circulation is inferred according to the type and location of connected ports and the information of components. Then, the connection pattern is retrieved in the pattern library. If the corresponding pattern is found in the library, parameters of all holes are calculated and the temporary of holes are generated. Once all the temporary bodies have been generated, interference and minimum wall thickness check is performed. For interference check algorithm, distances between the generated temporary bodies and existing ones is used to judge whether the designed circulation meets the design requirements. As shown in Fig. 6, if the corresponding pattern is not found or the designed circulation interferes with other circulation or the mounting hole of hydraulic element, MA-NSGA-II algorithm [4] is called to perform the path planning. Then the parameters of all holes of oil circuit are extracted and temporary bodies are generated. The detailed descriptions of MA-NSGA-II algorithm please refer to [4].

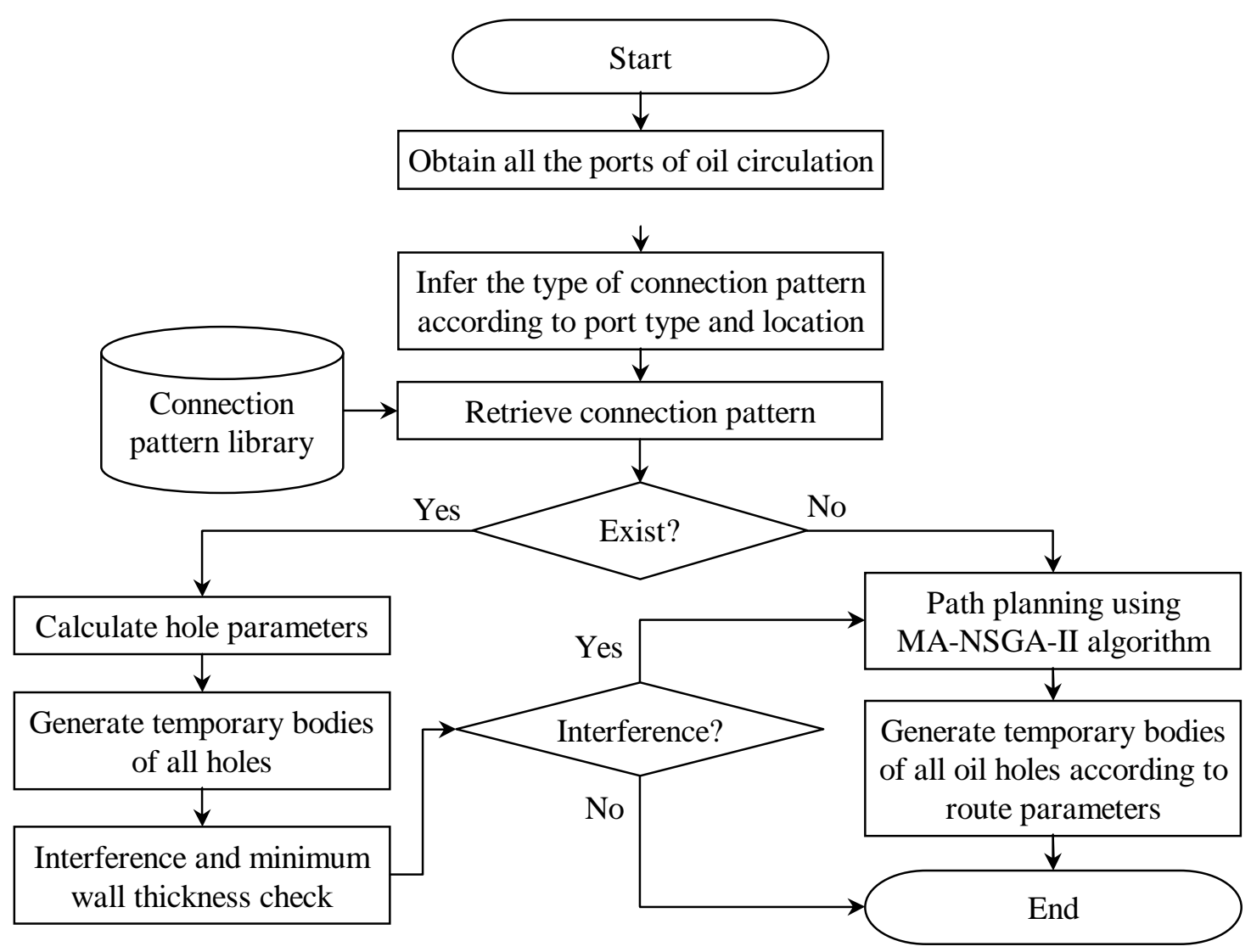

Fig. 6 Connection design process of oil circulation.

\section{Case study}

Fig. 7 illustrates a hydraulic scheme diagram including 12 hydraulic components and 7 oil circuits. The hydraulic components, oil circuits and connection relation of ports are listed in Table 1 and Table 2. Design sequence of oil circuits is P4, P2, P7, P1, P3, P5 and P6. The groups of components and component layout sequence are shown in Fig. 8. Reasoning results which include installation surface and location of components are listed in Table 2. Results of layout and connection design are shown in Fig. 9. Main circuits, include P1, P2, P3, P4 and P7, are designed by using pattern matching of typical oil circuits. Oil circuit P5 and P6 are designed by using intelligent path planning algorithm. As shown in Fig. 9, due to that the hole-arrangement of main circuits has been taken into full consideration in the component layout stage, design of main circuits is greatly simplified. 


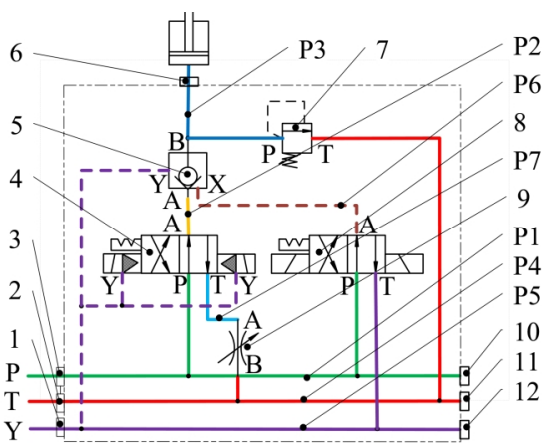

Fig. 7 Hydraulic schematic diagram.

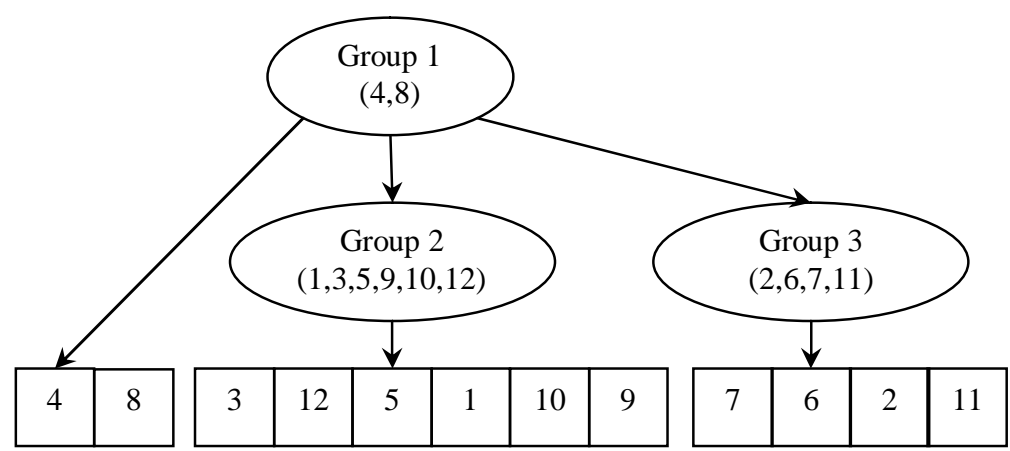

Fig. 8 Component grouping.

Table 1 Oil circuits information

\begin{tabular}{lll}
\hline Path ID & Diameter & Connection relation \\
\hline P1 & $34 \mathrm{~mm}$ & $3: \mathrm{IO} / 4: \mathrm{P} / 8: \mathrm{P} / 12: \mathrm{IO}$ \\
P2 & $38 \mathrm{~mm}$ & $4: \mathrm{A} / 5: \mathrm{A}$ \\
$\mathrm{P} 3$ & $32 \mathrm{~mm}$ & $5: \mathrm{B} / 7: \mathrm{P} / 6: \mathrm{IO}$ \\
$\mathrm{P} 4$ & $40 \mathrm{~mm}$ & $2: \mathrm{IO} / 9: \mathrm{B} / 7: \mathrm{T} / 11: \mathrm{IO}$ \\
P5 & $18 \mathrm{~mm}$ & $1: \mathrm{IO} / 5: \mathrm{Y} / 8: \mathrm{T} / 4: \mathrm{Y} / 10: \mathrm{IO}$ \\
P6 & $8 \mathrm{~mm}$ & $5: \mathrm{X} / 8: \mathrm{A}$ \\
P7 & $38 \mathrm{~mm}$ & $9: \mathrm{A} / 4: \mathrm{T}$ \\
\hline
\end{tabular}

Table 2 Information of components and mounting locations

\begin{tabular}{lllll}
\hline Components ID & Components & Mounting surface & Reference surface of HMB & Location(mm) \\
\hline 1 & SNO-A8-SR3-8 & 2 & $1 / 5$ & $200 / 60$ \\
2 & VF3005 & 2 & $1 / 5$ & $55 / 195$ \\
3 & VF3005 & 2 & $1 / 5$ & $170 / 115$ \\
4 & 4WEH32_6X & 5 & $1 / 2$ & $35 / 180$ \\
5 & SL30P-4X_P1 & 3 & $2 / 5$ & $130 / 165$ \\
6 & VF3002 & 3 & $2 / 5$ & $190 / 75$ \\
7 & DBDS10K 1X & 1 & $2 / 5$ & $195 / 155$ \\
8 & -WE6_6XE_K4 & 5 & $1 / 2$ & $155 / 220$ \\
9 & NDVP-30 & 1 & $2 / 5$ & $60 / 220$ \\
10 & VF3005 & 4 & $1 / 5$ & $170 / 115$ \\
11 & VF3005 & 4 & $1 / 5$ & $55 / 195$ \\
12 & SNO-A8-SR3-8 & 4 & $1 / 5$ & $200 / 60$ \\
\hline
\end{tabular}
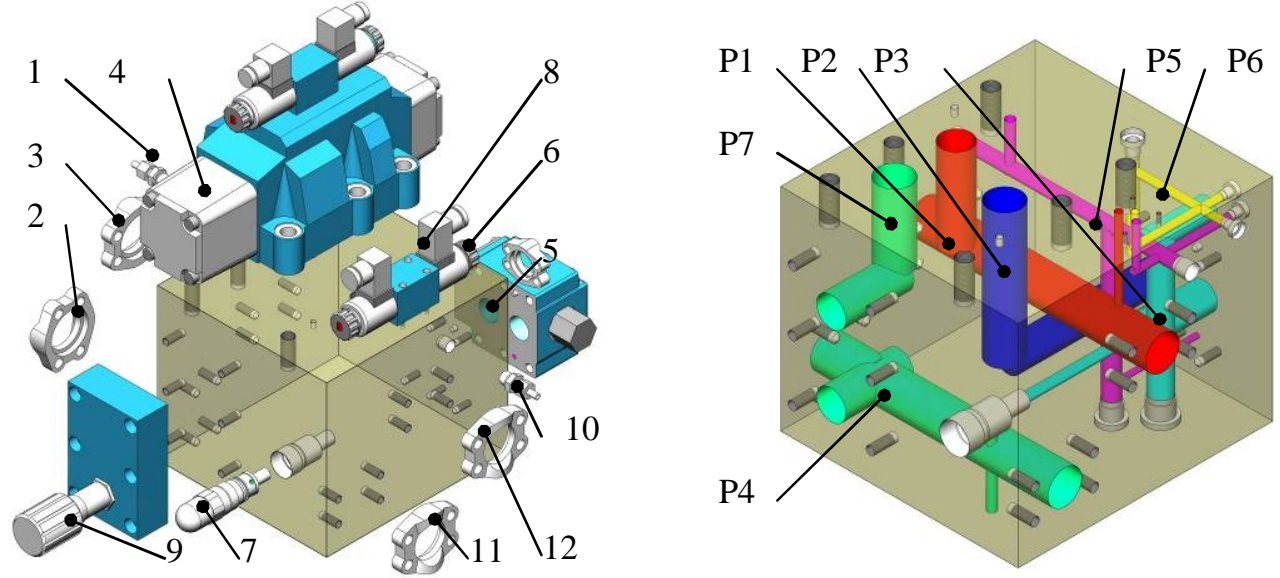

Fig. 9 Design result of hydraulic manifold block. 


\section{Conclusions}

The interactions between the external components layout and internal connection design are the major problem that greatly increase the design complexity of hydraulic manifold blocks. To solve the problem, an intelligent CAD system for the hydraulic manifold blocks was developed. Component layout rules and typical oil circuit connection pattern were proposed to integrate empirical knowledge, design expertise, design standard and code. A core components based component layout design method was proposed, which can reduce the design complexity of main oil circuits. A design approach for oil circulation was presented by combining the pattern matching of typical oil circulation and intelligent path planning algorithm, which can improve the design efficiency by effectively reusing design knowledge of oil circuits.

The feasibility of the proposed methodology and intelligent design system has been verified by a design example. Further work will be carried out to enrich the layout rules for hydraulic element and typical oil circuit patterns.

\section{Acknowledgements}

This work was financially supported by the National Natural Science Foundation of China (Grant Number: 51275340).

\section{References}

[1] R. Chambon and M. Tollenaere: Computer-Aided Design Vol. 23 (1991), p. 213

[2] F. Xu, D. Wang, Y. Gao and C. Jia: Machine Tool \& Hydraulics Vol. 36 (2008), p. 46 (In Chinese).

[3] H. Zhou: Research of theory and methods of hole optimization design of the hydraulic manifold block (Doctoral Dissertation, Shanghai Jiaotong University, Shanghai 2001) (In Chinese).

[4] G. Li: Research and application on modeling theory and optimization methodology for hydraulic manifold block (Doctoral Dissertation, Tianjin University, Tianjin 2012) (In Chinese).

[5] W. Liu, S. Tian, C. Jia and Y. Cao: Journal of Shanghai University Vol. 12 (2008), p. 261 (In Chinese).

[6] W. Zhang, H. Cheng, B. Lu, F. Guo and C. Ma: Journal of Nanjing University of Science and Technology Vol. 38 (2014), p. 354 (In Chinese).

[7] H. Cheng, W. Zhang, B. Lu, F. Guo and C. Ma: Computer Integrated Manufacturing System Vol. 20 (2014), p. 2376 (In Chinese).

[8] J. Bai and W. Niu: Chinese Journal of Engineering Design Vol. 21(2014), p. 589 (In Chinese). 